\title{
Preparation and Biological Activity of New Collagen Composites Part II: Collagen/Reduced Graphene Oxide Composites
}

\author{
Todorka G Vladkova, ${ }^{1,}$ Iliana A Ivanova, ${ }^{2}$ Anna D Staneva, ${ }^{1}$ Madalina G Albu, ${ }^{3}$ Ahmed S A Shalaby, ${ }^{1}$ \\ Tanya I Topousova, ${ }^{3}$ and Anelia S Kostadinova ${ }^{4}$ \\ ${ }^{1}$ University of Chemical Technology and Metallurgy, Sofia, Bulgaria \\ ${ }^{2}$ Biological Faculty, Sofia University “Saint Kliment Ohridski”, Bulgaria \\ ${ }^{3}$ Division Leather and Footwear Research Institute (ICPI), INCDTP, Bucharest, Romania \\ ${ }^{4}$ Institute of biophysics and biomedical investigations, BAS, Bulgaria \\ "Corresponding author: Todorka G Vladkova, 1University of Chemical Technology and Metallurgy, Sofia, Bulgaria. E-mail: tgv@uctm.edu
}

Received 2017 February 14; Revised 2017 February 28; Accepted 2017 February 28.

\begin{abstract}
With the idea of exploring the biological activity of some newly synthetized chemical compounds and their combinations for development of novel antimicrobial collagen biomaterials, a serial investigation was initiated, starting with the preparation and biological activity study of Collagen $/ \mathrm{ZnTiO}_{3}$ nano-composites. This serial investigation continued with the preparation and biological activity study of new collagen-based composites in which self-prepared reduced graphene oxide (RGO) sheets were included as an antimicrobial agent. The new porous collagen/RGO composites demonstrated specific antimicrobial activity to different types microbial species; well pronounced activity against Gram-positive microorganisms (Listeria innocua and Bacillus cereus, both bacteria with typical chains forming, large size cells, and Candida lusitaniae, fungus with specific micelle organization) and lack of activity against Gram-negative bacteria (Pseudomonas putida, Salmonella enterica, Pseudomonas aeruginosa, and Escherichia coli; all bacteria with small size cells) combined with lack of cytotoxicity to eukaryotic cells. For the first time, well-pronounced antifungal activity of collagen/RGO composites, depending on the RGO concentration was observed. Sterile zone of $17 \mathrm{~mm}$ was measured for C. lusitaniae on collagen/RGO composite, $2: 1 \mathrm{wt} / \mathrm{wt}$. The possible mechanism of the biological activity of the new collagen/RGO composites was correlated with their characteristics and the specific cell morphology and size of the test microorganisms. The results of this investigation demonstrated that with their specific and adjustable bioactivity, the new collagen/RGO composites are promising antimicrobial biomaterial for variety of biomedical applications, including tissue engineering.
\end{abstract}

Keywords: Collagen/ RGO Composites, Antibacterial and Antifungal Activity, Cytotoxicity

\section{Background}

Collagen is one of the natural polymers most frequently used in the development of medical devises with a variety of applications, including wound healing, tissue engineering, coatings, medical membranes, and others. The improved protection of most medical devices against infections is a significant current challenge. One of the easiest and most effective ways among the large variety of known approaches to add to the antimicrobial activity of biomaterials is the development of composites including antimicrobial agents. With the idea of exploring the biological activity of some newly synthetized chemical compounds, plant extracts, and their combinations for development of novel antimicrobial collagen biomaterials, a serial investigation was initiated, starting with the preparation and biological activity study of collagen $/ \mathrm{ZnTiO}_{3}$ nanocomposites (1).

This serial investigation was continued with preparation and biological activity study of new collagen-based composites, in which reduced graphene oxide (RGO) sheets were included as an antimicrobial agent.

During the past few years, different carbon materials, such as graphene, graphene oxide (GO) and reduced graphene oxide (RGO) were intensively studied as potential antimicrobial agents in tissue engineering biomaterials with minimal toxicity to mammalian cells. Their biocompatibility, high surface area, high mechanical strength, as well as ability to induce sustained stem cell growth and differentiation to various lineages are additional advantages (2).

Nano-sheets of both GO and RGO were reported to effectively inhibit the growth of $E$. coli showing simultaneously minimal cytotoxicity to mammalian cells. The toxicity to bacteria was suggested to be due to a membrane damage caused by rupture in contact with the particulate edges, which was confirmed by scanning electron microscopy (SEM) (3).

The first study of bacterial interaction with graphenelike surface was undertaken by Akhavan and Chaderi 
in 2010 (4). Gram-negative (Escherichia coli) and Grampositive (Staphylococcus aureus) bacteria as well as a single and few layers GO and RGO, deposited onto stainless steel substrate, with predominant particle edges exposure, were used in this investigation. A loss of viability for both E. coli and S. aureus was observed, which was more significant for the Gram-positive bacteria. Measurement of efflux of the cytoplasmic materials showed that membrane damage in contact with GO and RGO particles occurred, which supports the observed greater toxicity toward Gram-positive bacteria. The RGO surface had a greater ability to inhibit attachment and to kill bacteria, presumably due to its sharper edges than the GO counterparts (4).

Further investigation (5) was focused on the potential of E. coli "wrapping" with graphene nano-sheets in order to reduce its bioactivity. No significant inactivation of these Gram-negative bacteria was observed in presence of GO or RGO in suspension. However, the addition of melatonin (reducer) resulted in a functionalization of the graphene particles and aggregation of bacterial cells. Bacteria enclosed in GO or RGO particles, observed by Atomic Force Microscopy (AFM), were supposed to be the reason for the decrease in the amount of active cells in this case.

To better understand the antimicrobial mechanism, the antibacterial activity of 4 types graphene-based materials, graphite, graphite oxide, graphene oxide (GO), and reduced graphene oxide (RGO), toward Escherichia coli was compared. Based on the results of this investigation, a 3 step antimicrobial mechanism was proposed: i) initial cell deposition on the graphene-based materials, ii) membrane stress caused by the direct contact with the sharp nanosheets, and iii) ensuing superoxide anionindependent oxidation. Physicochemical properties such as density of functional groups, particles size of the carbon materials, and conductivity influenced their antibacterial activity (6).

Oxidative stress-mediated antibacterial activity of GO and RGO in Pseudomonas aeruginosa was also reported (7). The GO and RGO showed dose-dependent antibacterial activity against $P$. aeruginosa cells through the generation of reactive oxygen species, leading to cell death, which was confirmed through a resulting nuclear fragmentation.

Interactions of RGO particles in suspensions were a subject of many studies with comparable results to those involving substrates produced from these particles $(6,8)$.

The antibacterial efficiency of GO and RGO nano-sheets was studied against both Gram-positive (Enterococcus faecalis and Bacillus subtilis) and Gram-negative (E. coli and Salmonella typhimurium) bacteria by evaluation of the Minimum Inhibitory Concentration (MIC) of the particles (9). The MIC of the RGO was significantly lower for Gram- negative bacteria, hypothesized to be due to the much thinner peptidoglycan layer of these types of bacteria. This is in contrast to other studies, which suggest that the presence of a secondary cell membrane of Gram-negative bacteria provides a better resistance to membrane-induced damage in presence of RGO particles. It has also been reported that enhanced lipid peroxidation occurred in the suspensions containing RGO.

Thrombogenicity, biocompatibility, and cytotoxicity studies of RGO-modified acellular pulmonary valve tissue were reported to be connected with the current strategies of tissue engineering. No significant effect for RGO modified surfaces on the thrombogenicity and biocompatibility was observed as compared to the non-modified surface. $\mathrm{Cy}$ totoxicity indicated that the RGO can damage cells in direct contact, yet, it had no effect on the viability of fibroblasts in indirect contact (10).

A comparative in vitro and in vivo study (11) on the bioactivity of GO and RGO films, as well as of collagen scaffolds coated with GO and RGO, clearly demonstrated the higher biological activity of RGO- and RGO-coated collagen scaffolds, as detected by AFM and SEM observations, calcium absorption test, compression test and MC3T3-E1 cell seeding. Calcium absorption and alkaline phosphatase activity were strongly enhanced by RGO, suggesting that RGO is effective for osteogenic differentiation. The SEM showed that RGO-coated collagen scaffolds had rough and irregular surfaces. The compressive strengths of GO- and RGOcoated scaffolds were approximately 1.7-fold and 2.7-fold greater, respectively, when compared with the non-coated scaffold. All results suggested that the RGO-coated scaffolds are more bioactive than GO-coated scaffolds.

It was found that RGO-coated Hydroxyapatite (HAp) and other substrates stimulate spontaneous estrogenic differentiation of human mesenchyme stem cells that is of great interest for bone tissue engineering and regeneration $(12,13)$.

Among the carbon materials, RGO was most often used in composites and coating applications because its surface has a greater ability to inhibit attachment and to kill bacteria presumably due to its sharper edges than the other carbon material.

With their anti-bacterial activity, combined with unique physicochemical properties, biocompatibility as well as low both thrombogenicity and cytotoxicity to mammalian cells, RGO containing biomaterials hold significant potential for use in next-generation antimicrobial biomaterials and medical devices.

There is no consensus regarding RGO inherent antibacterial nature, yet, it is already known that it depends on some physical parameters like RGO sheets size and layer number both being determined by the preparation 
method and operation conditions. In the studies of bacterial interactions, bacteria with simple geometries were used such as spheres and rods, whereas other complex geometries were more common (14).

It is of major interest to expand knowledge on the great importance of clinical applications of microbes.

In addition, controversial results were reported about RGO antimicrobial activity toward Gram negative and Gram positive bacteria $(5,9)$.

So far, no study on collagen/RGO anti-fungal activity has been presented in the literature.

No reports were found about collagen/RGO composites and their biological activity against prokaryotic and eukaryotic cells.

Therefore, this study aimed at preparing new collagen composites with expected antimicrobial activity using selfprepared and characterized RGO sheets, and evaluating their biological activity against a variety of microbial cells with specific morphology and variety of eukaryotic cells.

\section{Methods}

\subsection{Preparation and Characterization of RGO}

The RGO used in this study (with less that $2 \%$ to 3\% wt impurity of graphite materials) was prepared by commonly-used chemical exfoliation method starting from purified natural graphite powder (99.9\%, Alfa Aesar Co.) and employing sodium borohydride as a reducing agent, as described previously (15).

The phase formation and structural transformation were detected by X ray phase analysis (Bruker D8 Advance, Germany; $\mathrm{Cu} \mathrm{K} \alpha$; LynxEye detector).

Transmission electron microscopy images were obtained using TEM JEOL 2100, at an accelerating voltage of $200 \mathrm{kV}$.

The microstructure and morphology of the crystalline products were studied by scanning electron microscopy (SEM-Jeol-357).

\subsection{Preparation of Collagen/RGO Composites}

Type I fibril collagen gel with concentration of $2.64 \mathrm{wt}$. percent was extracted from calf hide, using a previously described technology (16). The concentration of the collagen gel was adjusted to $1 \%$ and $\mathrm{pH}$ to 7.3 (that of the physiological medium), using $1 \mathrm{M}$ sodium hydroxide, while the antimicrobial agent (RGO powder) in 2:1, 2:0.8, 2:0.6, 2:0.4 or 2:0.2 ratios (wt/wt) was added. The collagen/antimicrobial agent composites prepared in this way were cross-linked with $0.5 \%$ glutaraldehyde (to dry collagen) at $40^{\circ} \mathrm{C}$ for 24 hours and then lyophilized at $-400^{\circ} \mathrm{C}$ to obtain porous (sponge) material using a Martin Christ freeze-dryer for 48 hours, as previously described $(17,18)$. Test samples with diameter of $9 \mathrm{~mm}$ were then prepared from each composite.

\subsection{SEM Observations Porous Collagen Composites}

The JEOL SEM, model JSM-5510, Japan apparatus was used to observe the morphological features of the studied porous collagen/RGO composites as well as of the RGO powder. The samples were gold-sputtered coated and viewed in the second electron mode with field emission gun.

\subsection{Compressive Modulus}

Uniaxial compression testing was performed to evaluate the effect of the incorporated collagen matrix RGO onto the modulus of the corresponding composite. Mechanical testing machine Instron, TT-DM, USA was employed to carry out the testing at a strain rate of $10 \%$ per minute. The results were average of testing of 6 identical samples.

\subsection{Antimicrobial Activity Testing}

Antimicrobial activity was tested for both, RGO dispersion and Coll/RGO composites.

The RGO dispersions were prepared in deionized water at varied concentrations of $0.5,5.0,50.0$, and $100 \mathrm{mg} / \mathrm{mL}$ by sonication in Bandelin apparatus for 15 minutes.

The microbial strains, including 4 Gram-negative bacteria (Salmonella enterica 2333, E. coli 264, Pseudomonas putida 1090, and Pseudomonas aeruginosa), 2 Gram-positive bacteria (S. epidermidis 3486 and Bacillus cereus 1095) as well as 2 fungi (Candida lusitaniae 74 - 4 and Saccharomyces cerevisiae) were used in this investigation, all provided from the national bank of microorganisms and cell cultures (NBIMCC), Bulgaria. They were cultured in media most suitable for each one. Escherichia coli 264 and S. enterica 2333 were grown in nutrient broth (NB Conda, Spain) at $37^{\circ} \mathrm{C}$ and $180 \mathrm{rpm}$ for 18 hours. Bacillus cereus 1095 and Staphylococcus epidermidis 3486 were propagated in nutrient agar and Candida lusitaniae 74 - 4 and S. cerevisiae in YGC, respectively, at $30^{\circ} \mathrm{C}$ and $120 \mathrm{rpm}$. Pseudomonas putida 1090 (ATCC 12633) was cultivated in synthetic liquid medium (ISO10712) at $22^{\circ} \mathrm{C}$ to $23^{\circ} \mathrm{C}$ and $180 \mathrm{rpm}$ for 12 hours. Microbial density of 0.5 to 0.8 was determined according to McFarland. The aliquots of $100-\mu \mathrm{L}$ microbial suspensions were randomly spread on solid medium (Nutrient agar - NA and YGC agar) and discs of investigated material were placed on them. The plates were left for 20 hours at $4^{\circ} \mathrm{C}$ to $60^{\circ} \mathrm{C}$ to afford diffusion of the nanoparticles followed by cultivation for 24 hours at $37^{\circ} \mathrm{C}, 30^{\circ} \mathrm{C}$, and $24^{\circ} \mathrm{C}$, respectively. The formed sterile zones around the disk samples were measured in $\mathrm{mm}( \pm 0.5)$.

These bacterial strains were used to test the antimicrobial activity of both. 


\subsection{Cytotoxicity Evaluation}

\subsubsection{Eukaryotic Cells}

Cytotoxicity was evaluated against 3 types of eukaryotic cells; model osteoblast cells, MG-63, 3T3 fibroblasts, and MDCK kidney epithelial cell line, all provided by the NBIMCC, Bulgaria. The used eukaryotic cells were maintained at standard conditions in humidified atmosphere with $5 \%_{2}$, at $37^{\circ} \mathrm{C}$, in the corresponding medium $\mathrm{F} 12$ or DMEM (SIGMA), supplemented with 10\% Fetal Bovine Serum (FBS) (BioWhittaker TM) and 1\% (v/v) antibioticantimitotic solution (penicillin $100 \mathrm{U} / \mathrm{mL}$, streptomycin $100 \mathrm{ug} / \mathrm{mL}$ and amphotericin B $0.25 \mathrm{ug} / \mathrm{mL}$, BioWhittaker ). For assessment of cytotoxicity, materials were embedded in 96-well plates and hydrated with $200 \mu \mathrm{L}$ culture medium for 12 to 24 hours. The cells were then seeded at a concentration of $1 \times 105$ cells/mL, and bright field microscopy pictures were taken after 24 hours, and cytotoxicity was evaluated by crystal violet test and microscopic observation of the cell morphology.

\subsubsection{Crystal Violet Staining and Microscopic Observation}

Crystal violet staining was performed with some modifications (19); the residual cell monolayer was washed with Phosphate-Buffered Saline (PBS) and fixed with 4\% paraformaldehyde in PBS for 15 minutes. Next, plates were washed with water, and $200 \mu \mathrm{L}$ of $0.1 \%$ crystal-violet solution was added to every well. After 20 minutes of incubation at room temperature, the plates were washed with water and the protein-bound dye (which is corresponding to the cell number) was solubilized with $200 \mu \mathrm{L}$ of $10 \%$ acetic acid. The values of optical density were read on a micro plate reader (EPOCH UV/VIS Spectrometer) at $570 \mathrm{~nm}$ wavelength and the number of vital cells was calculated as a percentage of their total amount.

\section{Results}

It is known that the antibacterial activity of the RGO sheets depends on the dimensions and number of the sheets as well as on their aggregation and the dimensions of the aggregates. Therefore, characterization of the prepared RGO was included in this study.

To keep the native biological activity of the collagen, the studied porous collagen/RGO composites were prepared by sol-gel cryogenic drying. The RGO loading levels varied, since its antimicrobial activity is known to be concentration dependent.

Four Gram-negative bacteria, 2 Gram-positive bacteria and 2 fungi were used for evaluation the antimicrobial activity of the RGO sheets in suspensions and the collagen/RGO composites, supposing that this effect will depend on the specific morphology of the microbial cells.
Large variety of possible potential applications of the studied new collagen/RGO composites was the reason for investigating their cytotoxicity against 4 types of eukaryotic cells.

\subsection{Characteristics of the Used RGO}

The RGO of this investigation was in form of multilayered sheets with inter-planar distance of $0.339 \mathrm{~nm}$ as calculated by selected area (RGO (002) peak, $20=26.2^{\circ}$, JCPDS 75-2078) of XRD pattern (Figure 1).

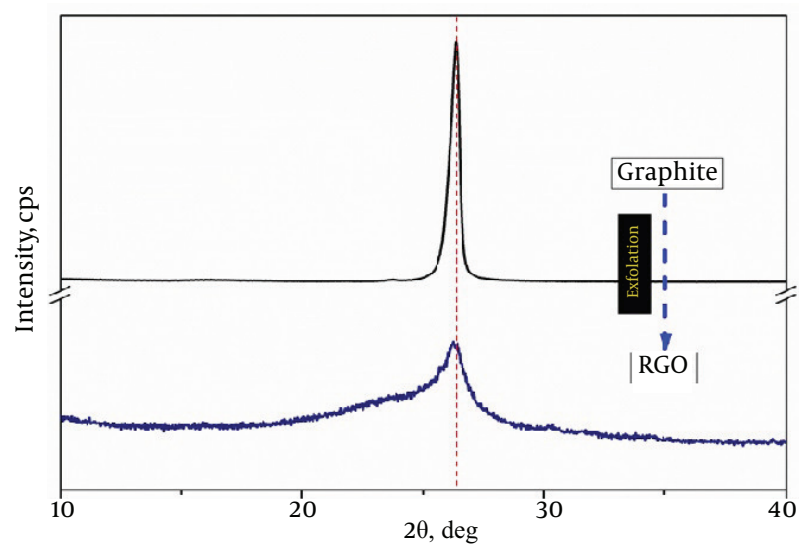

Figure 1. XRD Patterns of Graphite and Reduced Graphene Oxide Sample

The SEM images in Figure 2 present random aggregates of RGO sheets (A, B, and C) with distinct edges, wrinkled surfaces, and folding (D). The RGO sheets dimensions were of about $10 \times 20 \mu \mathrm{m}$.

The transmission electron microscopy (TEM) image in Figure 3 showed multi-layered RGO sheets. The layers were stacked on one another at lower resolution and the RGO sheet was transparent. The number of sheets was less than 5.

\subsection{Morphology of the Studied Porous Collagen/RCO Compos- ites}

Figure 4 shows the porous structure of a control collagen sample without RGO (Figure 4A and B), and collagen/RGO (2:1, wt/wt) composite (Figure 4C-F). The porous structure of the other collagen/RGO composites, less loaded with RGO (collagen: $\mathrm{RGO}=2$ : $0.8 ; 2$ : 0.6 and 2: $0.4 \mathrm{wt} / \mathrm{wt}$ ) was similar, and therefore the corresponding pictures are not presented here.

The photographs in this Figure show an open and interconnected relatively homogeneous porous structure of the control collagen sample (Figure 4A) and of the 

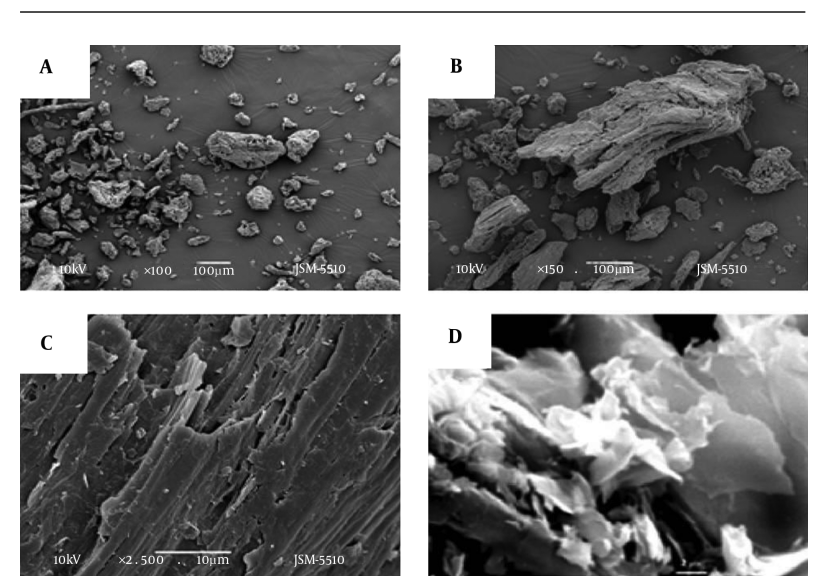

$\overline{\text { Figure 2. Scanning Electron Microscopy Images of Reduced Graphene Oxide Aggre- }}$ gates (A), (B) and Sheets (B), (D)

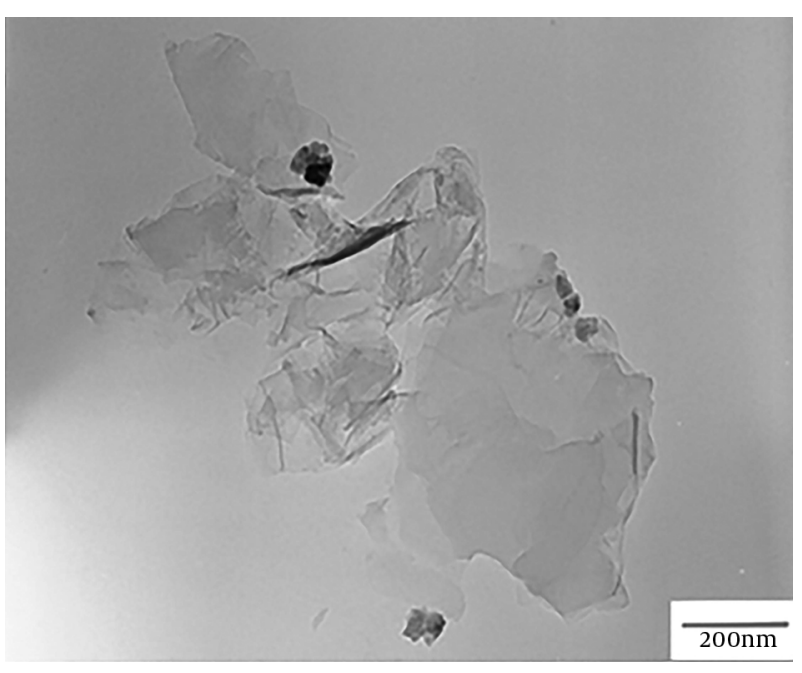

Figure 3. Transmission Electron Microscopy Image Showing Multi-Layered Reduced Graphene Oxide Sheets

studied collagen/RGO composites with relatively homogeneous distribution of RGO aggregates in the collagen matrix (Figure 4C). The RGO aggregates with different dimensions were wrapped in the collagen matrix, some of them partially covered by matrix collagen (Figure 4D-F).

\subsection{Compressive Modulus}

Expecting that the presence of RGO could influence the mechanical strength of the studied collagen/RGO composites, their compressive modulus at $10 \%$ deformation was estimated. The test results are presented in Table 1 . The compressive modulus of the 2 collagen/bioactive glass ceramic composites, prepared under the same conditions (by sol-gel cryogen drying and with the same collagen)
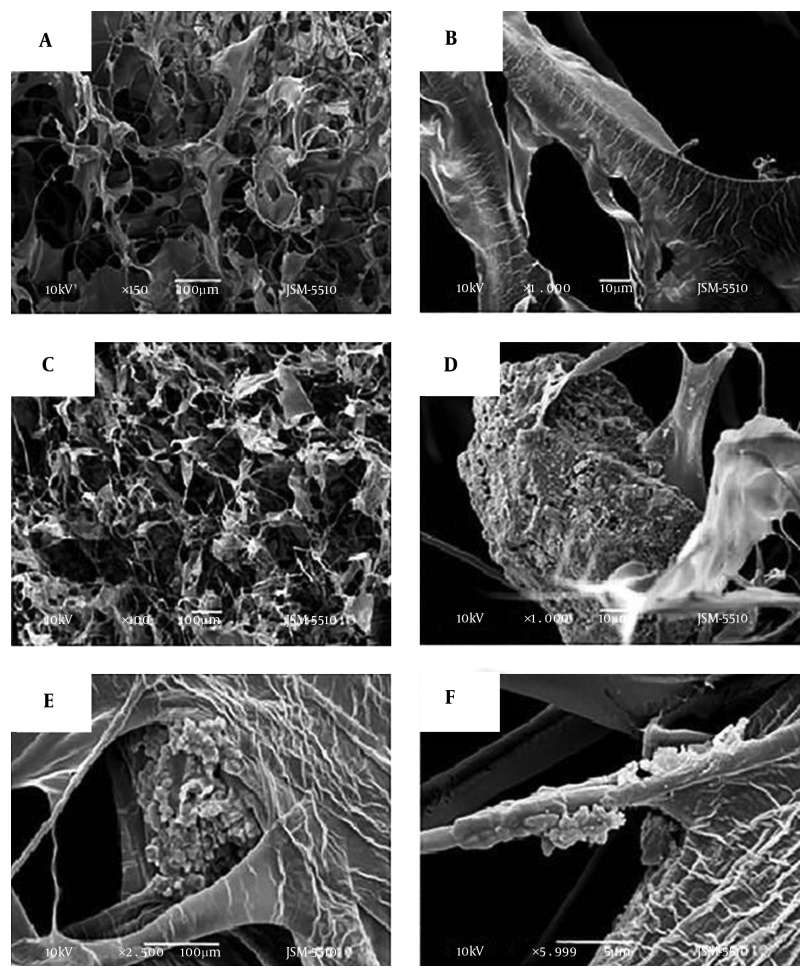

Figure 4. Scanning Electron Microscopy Images of Porous Collagen Matrix Without Reduced Graphene Oxide (A, B) and of Collagen/RGO composite, 2:1 wt/wt (C, D, E, F)

(20), is presented to compare the reinforcing effect of RGO to that of calcium phosphate silicate glass ceramic (CPS). As it was expected, the collagen/RGO composites demonstrated RGO concentration-dependent increased modulus, $\mathrm{M}_{10}$ (Table 1, samples 2, 3, and 4), as compared to that of the control collagen matrix (Table 1 , sample 1 ). This indicates that keeping the interconnected porous structure of the corresponding composite, RGO significantly increases its mechanical strength; this effect is most strongly expressed at highest RGO loading level (collagen: $\mathrm{RGO}=2.0$ : $1.0 \mathrm{wt} / \mathrm{wt}$ ). The increase of the modulus, $\mathrm{M}_{10}$ is almost 2fold: from $15.1 \mathrm{KPa}$ for the control collagen sample (sample 1) up to $29.3 \mathrm{KPa}$ for the Collagen/RGO composite (sample 2). The modulus, $M_{10}$ of this collagen/RGO composite (2.0: 1.0, wt/wt) is similar to that of Coll/CPS (1: 1, wt/wt) of 32.2 $\mathrm{KPa}$, although there is a significantly lower RGO loading level compared to that of CPS.

\subsection{Antimicrobial Activity of RGO Dispersion}

The antimicrobial activity of RGO distilled water dispersions with different concentrations of $0.5,5.0,50.0$, and $100.0 \mathrm{mg} / \mathrm{mL}$ was tested against 5 bacteria, including E. coli, B. cereus, Salmonella choleraesuis, P. aeruginosa, and S. epidermidis. No antimicrobial activity was detected for RGO at the 
Table 1. Compressive Modulus at $10 \%$ Deformation, $\mathrm{M}_{10}, \mathrm{KPa}$ of Porous Collagen and Collagen/Reduced Graphene Oxide (RGO) Composites ${ }^{\mathrm{a}}$

\begin{tabular}{lc}
\hline Sample & $\mathbf{M}_{\mathbf{1 0}}, \mathbf{K P a}$ \\
\hline 1. Collagen matrix & 15.1 \\
\hline 2. Collagen/RGO (2:1.0, wt/wt) & 29.3 \\
\hline 3. Collagen/RGO (2:0.8, wt/wt) & 26.6 \\
\hline 4. Collagen/RGO (2:0.6, wt/wt) & 23.9 \\
\hline 5. Coll/CPS (4:1, wt/wt) (20) & 15.4 \\
\hline 6. Coll/CPS (1:1, wt/wt) (20) & 32.2 \\
\hline $\begin{array}{l}\text { a Collagen/CPS (calcium phosphate silicate and CPS bioactive glass ceramic) } \\
\text { composites are included in the for for comparison. }\end{array}$
\end{tabular}

studied concentrations ( $0.5 \mathrm{mg} / \mathrm{mL}$ to $100 \mathrm{mg} / \mathrm{mL}$ ) against three test bacteria, including $S$. choleraesuis, $P$. aeruginosa, and $S$. epidermidis. This is in congruence with former investigations demonstrating lack of antimicrobial activity of RGO in dispersion (5).

Figure 5 demonstrates the antimicrobial activity of RGO against the other 2 bacteria, Gram-negative, E. coli and Gram-positive, B. cereus. The careful observation of the petri dishes shows lack of growth of both, E. coli (Figure 5A) and and B. cereus (Figure $5 \mathrm{~B}$ ) on the nutrient medium under the droplet of RGO dispersion with the highest concentration (of $100 \mathrm{mg} / \mathrm{ml}$ ), i.e. no bacterial growth in the contact area with the RGO dispersion. This suggests antimicrobial effect of RGO in contact with the bacteria.
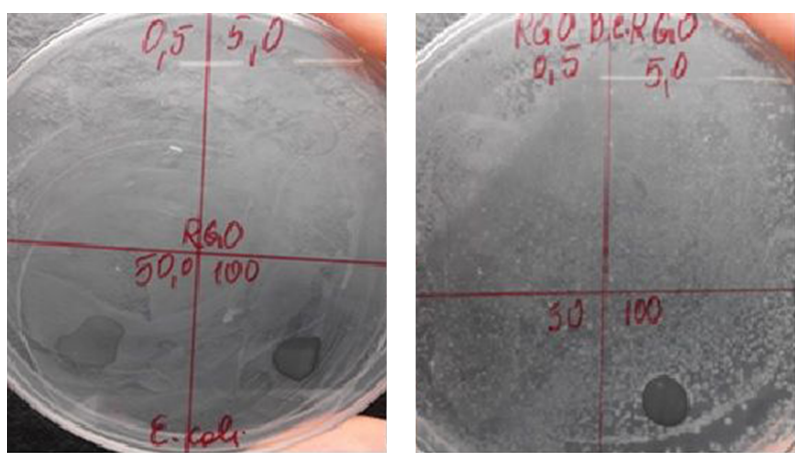

Figure 5. Antibacterial Effect of Distilled Water Reduced Graphene Oxide Dispersions With Concentrations of $0.5,5.0,50$ and $100 \mathrm{mg} / \mathrm{mL}$ against: (A) E. coli; (B) B. cereus

\subsection{Antimicrobial Activity of the Collagen/RGO Composites}

The antimicrobial activity of the studied collagen/RGO composites was tested against $4 \mathrm{Gram}$-positive (L. innocua, C. lusitaniae, S. epidermidis, and B. cereus) and 4 Gramnegative microorganisms ( $P$. putida, S. enterica, E. coli, and
P. aeruginosa). The corresponding results, as sterile zones in millimeters, are presented in Table 2 . It is evident that the studied microorganisms have different sensitivity to the included collagen matrix RGO sheets. Three (L.innocua, C. lusitaniae, and B. cereus) of the 4 tested Gram-positive microorganisms are sensitive to the included collagen matrix RGO; the first 2 being more sensitive. As it was expected, the antimicrobial activity is concentration dependent (Table 2, compare samples 1, 2, 3, 4, and 5). No sensitivity was indicated by the Gram-negative bacteria, including E. coli, S. enterica, $P$. aeruginosa, and P. putida.

It is interesting that Gram-positive, rod shaped, chainlike, test bacteria, L. innocua and B. cereus, was more sensitive to RGO sheets wrapped in collagen compared to S. epidermidis with spherical, coccoid shape.

For the first time antifungal activity was observed in the collagen matrix RGO. It is evident that the antifungal activity against $C$. lusitaniae is significant and dependent on the RGO concentration (Table 2, compared to samples 1 to 5$)$. This effect was strongly expressed at the 3 collagen/RGO composites with the highest concentration of RGO (ratios collagen/RGO = 2: $0.6 \mathrm{wt} / \mathrm{wt}$.). The sterile zone for these composites increases from $6.5 \mathrm{~mm}$ to $17.0 \mathrm{~mm}$. Very good activity was found against Gram-positive L. innocua (sterile zone of 12.8 to $17.0 \mathrm{~mm}$ ), at the same ratios.

\subsection{Cytotoxicity}

Three types of eukaryotic cells, most often used in tissue engineering, including osteoblasts (MG-63), fibroblast (3T3), and kidney epithelial (MDCK II) cells, were used to evaluate the cytotoxicity of the studied new antimicrobial collagen/RGO composites. Crystal violet assay was employed allowing quantification of cells viability, simultaneously with evaluation of the cell morphology.

The results of this test are presented in Table 3 and Figure 6 .

As seen from Table 3, the eukaryotic cells viability on the 24th hour of their seeding was high; about 100\% for fibroblast, 3T3, and the epithelial cells, MDCK, and less (about $70 \% \pm 20 \%$ ) for osteoblast, MG63, yet, still significant.

The morphology of the same cells on the 24 th hour of their seeding, presented in Figure 6, shows lack of dead cells of any employed cell line, including MG 63 that demonstrated less viability. The comparison of Figure 6D to Figure $6 \mathrm{C}$ as well as of Figure $6 \mathrm{~F}$ to Figure $6 \mathrm{E}$ showed no significant difference in the cell morphology on the collagen/RGO composite and in absence of this biomaterial for the used fibroblasts and epithelial cells, irrespectively. A comparison of Figure 6B to 6A for the morphology of osteoblasts, MG 63, is presented on collagen/RGO composite and in absence of this biomaterial, respectively demon- 
Table 2. Antimicrobial Activities of Collagen/ Reduced Graphene Oxide (RGO) Sponges, Loaded with Different Amounts of RGO, as a Sterile Zone (mm)

\begin{tabular}{|c|c|c|c|c|c|c|c|c|c|}
\hline \multirow[t]{2}{*}{ Sample No. } & \multirow[t]{2}{*}{ Coll: RGO (wt/wt) } & \multicolumn{8}{|c|}{ Sterile zone $^{\mathbf{a}}, \mathbf{m m}$} \\
\hline & & P.putida & S enterica & P. aeruginosa & E. coli & C. Lusitaniae ${ }^{\mathbf{b}}$ & L. innocua & S. epidermidis & B. cereus \\
\hline 1. & $2: 1.0$ & 0 & 0 & 0 & 0 & 17.0 & 17.0 & 0 & 3.6 \\
\hline 2. & $2: 0.8$ & 0 & 0 & 0 & 0 & 10.5 & 16.5 & 0 & 2 \\
\hline 3. & $2: 0.6$ & 0 & 0 & 0 & 0 & 6.5 & 12.8 & 0 & 1.9 \\
\hline 4. & $2: 0.4$ & 0 & 0 & 0 & 0 & 5.3 & 8.0 & 0 & . \\
\hline 5. & $2: 0.2$ & 0 & 0 & 0 & 0 & 1.2 & 7.0 & 0 & 0 \\
\hline
\end{tabular}
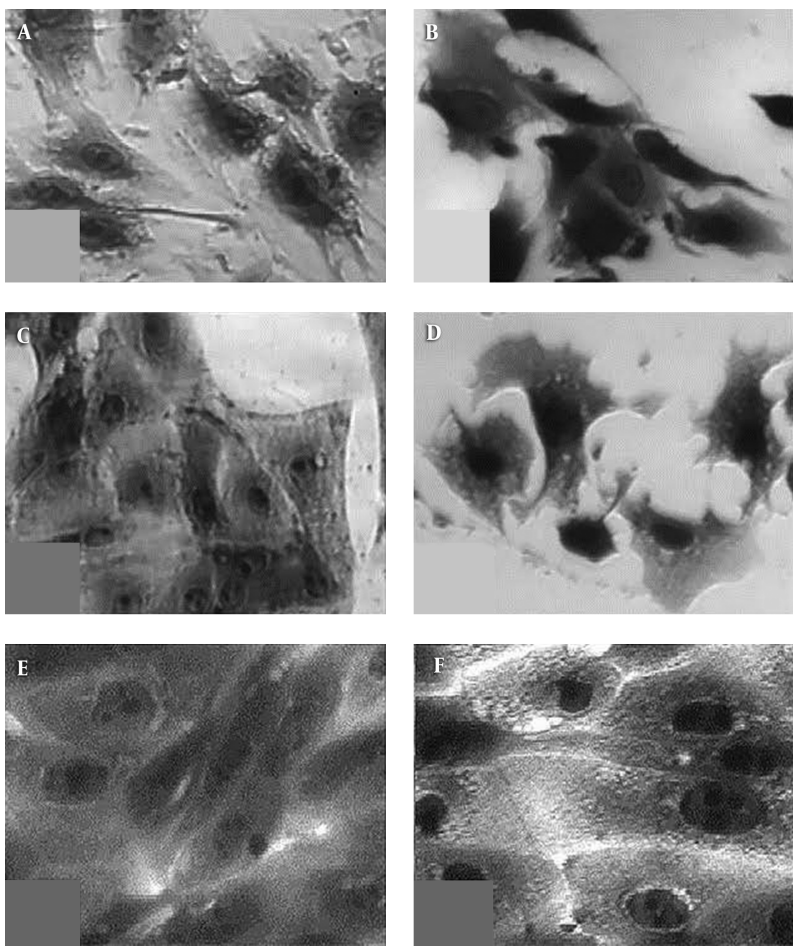

Figure 6. Cell Morphology in Polystyrene Plate Wells (A, C, E) and on Collagen/ Reduced Graphene Oxide Composite, 2:1 wt/wt (B, D, F) of osteoblasts, MG63 (A, B), fibroblasts, 3T3 (C, D) and kidney epithelial cells, MDCK II (E, F); magnification 100x

Table 3. Crystal Violet Assay of Eukaryotic Cells: Osteoblast, MG63, Fibroblast, 3T3 and Kidney Epithelial, MDCK II on Collagen/ Reduced Graphene Oxide Composite, $2: 1 \mathrm{wt} / \mathrm{wt}$

\begin{tabular}{lc}
\hline Eukaryotic Cells & Cell Viability, \% \\
\hline MG 63 & $70 \pm 20$ \\
3 T3 & $100 \pm 8$ \\
MDCK II & $96 \pm 7$ \\
\hline
\end{tabular}

strate that the cells on the collagen/RGO composite are well spread but still not enough dence placed each to other.
Some lamellipodia are observed showing that some of the cells are moving to the other cell aggregates, i.e. it could be expected that during a longer incubation time, confluent cell mono-layer will be formed. In fact, as it was expected, specific response of the used different type eukaryotic test cells was observed, without any apoptotic morphology or other stages of cell death during 24 hours of incubation. This gives reason to accept that the studied new collagen/RGO composites are non-toxic for eukaryotic cells.

\section{Discussion}

In contrary to the literature that RGO in suspensions does not demonstrate antibacterial activity, we found that its high concentrated $(100 \mathrm{mg} / \mathrm{mL})$ dispersion suppresses the development of 2 bacteria, Gram-negative E. coli and Gram-positive B. cereus. Consequently, not enough concentration for RGO dispersions could be a reason for the lack of antibacterial activity in some cases, because the corresponding microbial cells in fact enter the water medium without the need to meet RGO sheets.

The RGO used in this study consists of multilayer (up to 5) sheets with relatively large area (up to about $10 \times 20 \mu \mathrm{m}$ ) that tend to aggregate. They were dispersed in a collagen gel to form porous collagen/RGO composite after cryogen drying; the last one keeping the native biological activity of the collagen. No chemical interactions between RGO and collagen matrix were expected under these conditions. Aggregates of RGO sheets were wrapped in the collagen matrix, some of them partially coated by matrix collagen, as depicted by SEM images.

Although the mechanism of the antimicrobial activity of RGO is not fully understood, it is generally accepted that it includes an effect of direct cell membrane contact with sharp RGO nano-sheets (4). In addition, destructive extraction of large amount phospholipids from E. coli cell membrane by graphene nano-sheets (due to strong dispersion interactions between RGO and lipid molecules) is shown as 
a reason for the antibacterial activity of the graphene nanosheets (21).

The antimicrobial activity of the new collagen/RGO composites, observed in this study was specific to different microbial organisms; pronounced against 3 of the 4 Grampositive microorganisms, 2 bacteria and 1 fungus, and no activity against the 4 Gram-negative bacteria used in this study. It could be suggested that the different sensitivity of the microbial cells to the new collagen/RGO composites is somehow connected to the characteristics of both the selfprepared multi-layer RGO sheets (size, aggregation, and number of the layers), and the collagen/RGO composites (pore size, distribution and way of attachment of RGO sheets and their aggregates) as well as the specific morphology of the test microbial organisms. The size of the interconnected pores of the collagen sponges with wrapped RGO sheets (and their aggregates) allows penetration of all test microbial organisms in this biomaterial.

In the SEM images of the new Coll/RGO composites, some agglomeration of the reduced graphene oxide particles as well as partial coverage of their sharp edges by matrix collagen was observed. The pronounced activity against Gram-positive bacteria L. innocua and B. cereus, both with typical, chains forming morphology and large size cells, could be a result of entailment of their filaments to the wrapped in the collagen matrix RGO and mechanical blockage.

Strongly expressed RGO activity against fungus was observed for the first time in this investigation. This activity against the test fungus $C$. lusitaniae that has specific micelle organization, can be explained by a similar direct interaction of its chitin cell walls with the wrapped collagen matrix RGO sheets. Presenting in the nutrient medium antibiotic, chloramphenicol $(0.1 \mathrm{~g} / \mathrm{L})$ probably assists the antifungal action of the collagen/RGO composites. The chloramphenicol structure is similar to that of phenylpyrroles, known to have antifungal activity against the filamentous fungi Candida lusitaniae and especially sho1Delta mutants (22). The assisting action of the chloramphenicol could be assumed although the Candida lusitaniae strain used in this study was not a mutant (23).

The lack of antibacterial activity against Gram-negative and Gram-positive bacteria, like E. coli and S. epidermidis, both having spherical shape and small dimensions, is presumably because of their easy penetration in the collagen/RGO composites (without contact with the wrapped RGO sheets) or "slipping" on the collagen coated RGO sheets without mechanical damage of the cell walls and membranes.

All 3 tested eukaryotic cell lines, used in this investigation, originated from tissues where in vivo they were in contact with different types of collagen with different density:
MDCK II are epithelial kidney cells that require the presence of the basal lamina; fibroblasts such as 3T3 cells, usually build up the connective tissue; collagen is main protein of bones among other tissues. As a natural environment of the fibroblasts and osteoblasts, the collagen, presented in the studied composites attracts the eukaryotic cells. This is probably the main reason for cell adhesion. The RGO sheets, wrapped in the collagen matrix and partially covered by matrix collagen do not influence the eukaryotic cells vitality. More in depth studies of these mechanisms will support the development of biomaterials with improved bioactivity.

\subsection{Conclusions}

New antimicrobial porous collagen/RGO biomaterials were developed that have well pronounced activity against both Gram-positive bacteria and fungi, as the last one were detected for the first time. Sterile zone of $17 \mathrm{~mm}$ was measured for C. lusitaniae on collagen/RGO composite, 2:1 $\mathrm{wt} / \mathrm{wt}$.

The different antimicrobial activity of the new collagen/RGO composites towards Gram-positive and Gramnegative test microorganisms was supposed to be due to their specific morphology and size. The Gram-positive microorganisms, L. innocua and B. cereus had typical chains forming large size cells, while the fungus $C$. lusitaniae, with specific micelle organization, was possibly mechanically blocked by entanglement from the wrapped collagen matrix RGO sheets during their penetration in the pores of the Collagen/RGO composite that explains their sensitivity to the Collagen/RGO composites. The Gram-negative bacteria, P. putida, S. enterica, P. aeruginosa, and E. coli, all with spherical shape and small size cells, easily penetrate in the porous material without entanglements and hence they are not sensitive to the RGO.

Concentration-dependent reinforcement effect of RGO was indicated that offers a possibility to adjust the mechanical strength of the Collagen/RGO composites to the requirements of the corresponding tissue engineering biomaterial.

The results of this investigation demonstrate that with their specific and adjustable bioactivity and lack of cytotoxicity, the new collagen/RGO composites are promising antimicrobial biomaterials for a variety of biomedical applications, including tissue engineering.

\section{Acknowledgments}

Bulgarian Scientific Fund is gratefully acknowledged for their financial support of this investigation (grand DDVU 02/100/2010) that was performed in the frame of 
COST Action TD1305 "Improved Protection of Medical Devices Against Infections".

\section{References}

1. Albu MG, Vladkova TG, Ivanova IA, Shalaby AS, Moskova-Doumanova VS, Staneva AD, et al. Preparation and Biological Activity of New Collagen Composites, Part I: Collagen/Zinc Titanate Nanocomposites. Appl Biochem Biotechnol. 2016;180(1):177-93. doi: 10.1007/s12010-016-2092-x. [PubMed: 27138724].

2. Ding X, Liu H, Fan Y. Graphene-Based Materials in Regenerative Medicine. Adv Healthc Mater. 2015;4(10):1451-68. doi: 10.1002/adhm.201500203. [PubMed: 26037920].

3. Hu W, Peng C, Luo W, Lv M, Li X, Li D, et al. Graphene-based antibacterial paper. ACS Nano. 2010;4(7):4317-23. doi: 10.1021/nn101097v. [PubMed: 20593851].

4. Akhavan O, Ghaderi E. Toxicity of graphene and graphene oxide nanowalls against bacteria. ACS Nano. 2010;4(10):5731-6. doi: 10.1021/nn101390x. [PubMed: 20925398]

5. Akhavan O, Ghaderi E, Esfandiar A. Wrapping bacteria by graphene nanosheets for isolation from environment, reactivation by sonication, and inactivation by near-infrared irradiation. $J$ Phys Chem $B$. 2011;115(19):6279-88. doi: 10.1021/jp200686k. [PubMed: 21513335].

6. Liu S, Zeng TH, Hofmann M, Burcombe E, Wei J, Jiang R, et al. Antibacterial activity of graphite, graphite oxide, graphene oxide, and reduced graphene oxide: membrane and oxidative stress. ACS Nano. 2011;5(9):6971-80. doi: 10.1021/nn202451x. [PubMed: 21851105].

7. Gurunathan S, Han JW, Dayem AA, Eppakayala V, Kim JH. Oxidative stress-mediated antibacterial activity of graphene oxide and reduced graphene oxide in Pseudomonas aeruginosa. Int J Nanomedicine. 2012;7:5901-14. doi: 10.2147/IJN.S37397. [PubMed: 23226696].

8. Sreeprasad TS, Maliyekkal MS, Deepti K, Chaudhari K, Xavier PL, Pradeep T. Transparent, luminescent, antibacterial and patternable film forming composites of graphene oxide/reduced graphene oxide. ACS Appl Mater Interfaces. 2011;3(7):2643-54. doi: 10.1021/am200447p. [PubMed: 21688808].

9. Krishnamoorthy K, Veerapandian M, Zhang LH, Yun K, Kim SJ. Antibacterial efficiency of graphene nanosheets against pathogenic bacteria via lipid peroxidation. J Phys Chem C. 2012;116(32):17280-7. doi: 10.1021/jp3047054.

10. Wilczek P, Major R, Lipinska L, Lackner J, Mzyk A. Thrombogenicity and biocompatibility studies of reduced graphene oxide modified acellular pulmonary valve tissue. Mater Sci and Eng: C. 2015;53:310-21. doi: 10.1016/j.msec.2015.04.044.

11. Kanayama I, Miyaji H, Takita H, Nishida E, Tsuji M, Fugetsu B, et al. Comparative study of bioactivity of collagen scaffolds coated with graphene oxide and reduced graphene oxide. Int J Nanomedicine. 2014;9:3363-73. doi: 10.2147/IJN.S62342. [PubMed: 25050063].

12. Lee JH, Shin YC, Jin OS, Kang SH, Hwang YS, Park JC, et al. Reduced graphene oxide-coated hydroxyapatite composites stimulate spontaneous osteogenic differentiation of human mesenchymal stem cells. Nanoscale. 2015;7(27):11642-51. doi: 10.1039/c5nr01580d. [PubMed: 26098486].

13. Dubey N, Bentini R, Islam I, Cao T, Castro Neto AH, Rosa V. Graphene: A Versatile Carbon-Based Material for Bone Tissue Engineering. Stem Cells Int. 2015;2015:804213. doi: 10.1155/2015/804213. [PubMed: 26124843].

14. Notley SM, Crawford RJ, Ivanova EP. Bacterial interaction with graphene particles and surfaces. Adv Graphene Sci. 2013.

15. Shalaby A, Nihtianova D, Markov P, Staneva AD, Iordanova RS, Dimitriev YB. Structural analysis of reduced graphene oxide by transmission electron microscopy. Bulg Chem Comm. 2015;47(1):291-5.

16. Albu MG. Collagen gels and matrices for biomedical applications. Saarbrücken: Lambert Academic Publishing; 2011

17. Albu MG, Deselnicu V, Ioannidis I, Deselnicu D, Chelaru C. Chemical functionalization and stabilization of type I collagen with organic tanning agents. Korean J of Chem Eng. 2014;32(2):354-61. doi: 10.1007/s11814-014-0197-x.

18. Albu MG, Ghica MV. Spongious collagen-minocycline delivery systems. Farmacia. 2015;63(1):20-5.

19. Moskova-Doumanova V, Miteva G, Dimitrova M, Topouzova-Hristova T, Kapchina V. Methanol and chloroform extracts fromlamium albuml. Affect cell properties of A549 cancer lung cell line. Biotechnol Biotechnol Equip. 2014;26(sup1):120-5. doi: 10.5504/50yrtimb.2011.0022.

20. Albu M, Radev L, Titorenku I, Vladkova T. Fibrillar collagen/bioactive calcium phosphate silicate glass-ceramic composites for bone tissue engineering. Current Tissue Engineering. 2013;2(2):119-32. doi: 10.2174/22115420113029990010.

21. Tu Y, Lv M, Xiu P, Huynh T, Zhang M, Castelli M, et al. Destructive extraction of phospholipids from Escherichia coli membranes by graphene nanosheets. Nat Nanotechnol. 2013;8(8):594-601. doi: 10.1038/nnano.2013.125. [PubMed: 23832191].

22. Boisnard S, Ruprich-Robert G, Florent M, Da Silva B, Chapeland-Leclerc F, Papon N. Role of Sho1p adaptor in the pseudohyphal development, drugs sensitivity, osmotolerance and oxidant stress adaptation in the opportunistic yeast Candida lusitaniae. Yeast. 2008;25(11):849-59. doi: 10.1002/yea.1636. [PubMed: 19061190].

23. Kujumdzieva-Savova AV, Kechlibarova LI, Savov VA, Nedeva TS, Georgieva EI. Dependence of Candida lusitaniae 74-4 protoplast yield on the growth phaseand nutrient medium type. Dokladi B Lgarskata Akad Nauk. 1992;45(5):91-4. 